\title{
Low-Dimensional Self-Bound Quantum Rabi-Coupled Bosonic Droplets
}

\author{
Emerson Chiquillo \\ Escuela de Física, Universidad Pedagógica y Tecnológica de Colombia (UPTC), \\ Avenida Central del Norte, 150003 Tunja, Colombia
}

\begin{abstract}
We analytically calculate the leading quantum corrections of the ground-state energy of two- and one-dimensional weakly interacting Rabi-coupled Bose-Bose mixtures in the frame of the Bogoliubov approximation. We show that to repulsive intraspecies and attractive interspecies interactions, the effect of quantum fluctuations favors the formation of self-bound droplets. These liquidlike states are crucially affected by the Rabi coupling, leading thus to the appearance of a quantum instability. We derive meaningful formulas to describe the droplet phase in the one-dimensional case.
\end{abstract}

Introduction. In the last few years, self-bound states in ultracold and ultradilute gases beyond the mean-field approximation have attracted the attention providing new opportunities for experimental and theoretical research. In the pioneering theoretical works $[1,2]$, the quantum fluctuations have been proposed as the main stabilization mechanism of such self-bound structures in BoseBose mixtures, revealing the crucial role played by quantum many-body effects. This stabilization lies in the balance of the mean-field contribution, close to the collapse threshold, and the first quantum correction coming from the zero-point motion of the Bogoliubov excitations, allowing thus the birth of a new phase of matter, called liquidlike quantum droplet. Soon after this prediction, and in a different context, droplets in dipolar gases of atoms of ${ }^{164} \mathrm{Dy}$ [3-6] and ${ }^{168} \mathrm{Er}[7]$ were discovered. These intriguing breakthroughs reveal that strongly bosonic gases of magnetic atoms do not necessarily collapse, as previously assumed from a purely mean-field viewpoint. Instead, they get into a dipolar droplet phase. More recently, the predictions in Ref. [1] have been experimentally achieved with ultracold and dilute mixtures of gases of ${ }^{39} \mathrm{~K}$ atoms [8-10]. From a theoretical viewpoint, such a droplet phase remains ultradilute and weakly interacting, allowing for a perturbative description [11, 12]. In order to explain the experimental results obtained with dipolar Bose gases, a generalized time-dependent nonlocal Gross-Pitaevskii equation has been solved [13-18]. There have also been numerical works [19-21]. In the study of the leading quantum corrections in Bose-Bose mixtures, works employing Monte Carlo methods have also been carried out $[22,23]$. Thermal corrections on mixtures of two-component bosonic gases with short-range interactions have been studied in Ref. [24].

On the other hand, in the last decade, laser beams have been used to induce artificial transitions among different atomic hyperfine states $[25,26]$. In the framework of the mean-field approximation, extensive research was addressed to understand the properties of these synthetic non-Abelian gauge fields in neutral bosonic mixtures of ultracold gases [27-29]. These works have opened the door to a fascinating and fast development of phenomena with spin-orbit- and Rabi-coupled ultracold atoms [30]. Recently, a connection between the synthetic Rabi coupling and the quantum droplets in a three-dimensional
(3D) two-component Bose gas of interacting alkali-metal atoms was investigated in Ref. [31]. However, the liquidlike droplet phase is more ubiquitous and remarkable in low-dimensional Bose-Bose mixtures, as predicted in Ref. [2]. Thus these systems have given rise to great experimental and theoretical interest. Then, for the best understanding of the behavior of interacting pseudospinor bosonic systems in a lower dimensionality, it is important to analyze the quantum effect induced by the Rabi coupling.

In this Rapid Communication, motivated by the enhanced role of beyond-mean-field effects in ultracold gases, and the fast development of artificial couplings between atomic internal states, we address theoretically the formation and stability of self-bound liquidlike droplets in low-dimensional Rabi-coupled ultracold bosonic atoms. We consider two- and one-dimensional two-component mixtures, and we focus on the interesting case where the intraspecies interactions are weakly repulsive and the interspecies ones are weakly attractive. We obtain the conditions of formation of self-bound droplets in terms of the Rabi coupling and the strength of the interactions. Remarkably, we find that in both two- and one-dimensional scenarios, there is a critical Rabi frequency beyond which the self-bound droplet becomes unstable. In the one-dimensional (1D) case, and by considering a small Rabi-coupling regime, we obtain meaningful analytical formulas to describe the ground state of the droplet phase. It is also relevant to stress that such a 1D instability is similar to the collapse in a 3D Bose-Einstein condensate (BEC). At equilibrium some relevant quantities are also calculated, such as the chemical potential and speed of sound.

Rabi-coupled bosons. Consider the path-integral formalism for two interacting and equal-mass Rabi-coupled bosonic species with hyperfine states $(\uparrow, \downarrow)$, and governed by the action

$$
\begin{aligned}
S\left[\Psi, \Psi^{*}\right] & =\sum_{\alpha}\left[\psi_{\alpha}^{*}\left(\hbar \frac{\partial}{\partial \tau}-\frac{\hbar^{2}}{2 m} \nabla^{2}-\mu\right) \psi_{\alpha}\right. \\
& +\frac{1}{2} \sum_{\sigma} g_{\alpha \sigma}\left|\psi_{\alpha}\right|^{2}\left|\psi_{\sigma}\right|^{2}-\hbar \omega_{R}\left(\psi_{\uparrow}^{*} \psi_{\downarrow}-\psi_{\downarrow}^{*} \psi_{\uparrow}\right)(1)
\end{aligned}
$$

where we have used the shorthand notation $\mathbb{E} \equiv$ $\int_{0}^{\hbar \beta} d \tau \int_{L^{D}} d^{D} r \sum_{\alpha}, \beta=1 / k_{B} T, k_{B}$ is the Boltzmann 
constant, $D=1,2$, and $\alpha, \sigma=\uparrow, \downarrow$. Given the pseudospinor $\Psi=\left(\psi_{\uparrow}, \psi_{\downarrow}\right)^{T}$, each component is described by a complex bosonic field $\psi_{\alpha}(\mathbf{r}, \tau), \psi_{\sigma}(\mathbf{r}, \tau)$. Interaction effects will be taken into account through the intraand interspecies coupling constants $g_{\alpha \alpha}$ and $g_{\alpha \sigma}$, respectively. These couplings are related to the $s$-wave scattering lengths $a_{\alpha \alpha}$ and $a_{\alpha \sigma}$, which depend on the dimensionality of the system as discussed later. Transitions between the two states are induced by an external coherent Rabi coupling of frequency $\omega_{R}$. Due to the Rabi mixing between the states, only the total number of particles is conserved [32]. Thus it is assumed that the two components are in a state of chemical equilibrium $\mu$, where the chemical potential for the two components is the same $[33,34]$.

In order to calculate the ground state of the mixture we obtain the grand potential $\Omega=-\beta^{-1} \ln \mathcal{Z}$, where $\mathcal{Z}=\int \mathcal{D}\left[\Psi, \Psi^{*}\right] \exp \left(-S\left[\Psi, \Psi^{*}\right] / \hbar\right)$. To this end, we consider the superfluid phase, where a U(1) gauge symmetry of each component is spontaneously broken. Then we can set $\psi_{\alpha}(\mathbf{r}, \tau)=\sqrt{n_{\alpha}}+\eta_{\alpha}(\mathbf{r}, \tau)$, where $\sqrt{n_{\alpha}}$ corresponds to the macroscopic quasicondensate (mean-field approximation), and $n_{\alpha}=\left|\psi_{\alpha}\right|^{2}$ is the two- or one-dimensional quasicondensate density. Although strictly Bose-Einstein condensation is prevented in low dimensionality, a finitesize system at a sufficiently low temperature allows for a quasicondensation $[35,36]$. The Gaussian fluctuations around $\sqrt{n_{\alpha}}$ are given by $\eta_{\alpha}(\mathbf{r}, \tau)$. So, by expanding the action up to the second order in $\eta_{\alpha}(\mathbf{r}, \tau)$ and $\eta_{\alpha}^{*}(\mathbf{r}, \tau)$ [37], we arrive at the beyond-mean-field grand potential $\Omega\left(\mu, \sqrt{n_{\uparrow}}, \sqrt{n_{\downarrow}}\right)=\Omega_{0}\left(\mu, \sqrt{n_{\uparrow}}, \sqrt{n_{\downarrow}}\right)+\Omega_{g}\left(\mu, \sqrt{n_{\uparrow}}, \sqrt{n_{\downarrow}}\right)$ [38]. Here, $\Omega_{0}$ gives the mean-field contribution, while $\Omega_{g}$ takes into account the Gaussian fluctuations at zero temperature. Since we want $\sqrt{n_{\alpha}}$ to describe the quasicondensate, then in the action, the linear terms in the fluctuations vanish such that $\sqrt{n_{\alpha}}$ really minimizes the action [39]. Thus the mean-field approximation is obtained by minimizing $\Omega_{0}$ with respect to $\sqrt{n_{\alpha}}$. To get solutions from this condition, we use equal interspecies coupling constants $g_{11}=g_{22}=g$. In this way, two possible ground states are obtained, a symmetric and a polarized one [34]. Hereafter, we focus on the symmetric configuration with $n_{1}=n_{2}=n / 2$. So, we find the relation between the variational parameter $n$ and the chemical potential $\mu$, such that $n=2 \mu_{R} / g_{+}$, where $\mu_{R}=\mu+\hbar \omega_{R}$, $g_{+}=g(1+\epsilon)$, and $\epsilon=g_{\uparrow \downarrow} / g$. In that case, the mean-field grand potential simplifies to [31]

$$
\frac{\Omega_{0}}{L^{D}}=-\frac{\mu_{R}^{2}}{g_{+}}
$$

The contribution of the zero-temperature Gaussian fluctuations for the symmetric ground-state and equal interspecies strengths reads

$$
\frac{\Omega_{g}}{L^{D}}=\frac{1}{2} \frac{S_{D}}{(2 \pi)^{D}} \int_{0}^{\infty} d k k^{D-1}\left[E_{a}(k, \mu)+E_{b}(k, \mu)\right]
$$

with $S_{D}=2 \pi^{D / 2} / \Gamma(D / 2)$, the Rabi-bosonic excitations $E_{a}=\left[\varepsilon(k)\left[\varepsilon(k)+2 \mu_{R}\right]\right]^{1 / 2}$, and $E_{b}=\{\varepsilon(k)[\varepsilon(k)+$ $\left.\left.2 \bar{A}\left(\mu, \omega_{R}, \epsilon\right)\right]+\bar{B}\left(\mu, \omega_{R}, \epsilon\right)\right\}^{1 / 2}$, where we have the freeparticle energy $\varepsilon(k)=\hbar^{2} k^{2} / 2 m, \bar{A}=\mu_{R} \Delta+2 \hbar \omega_{R}$, $\bar{B}=4 \hbar \omega_{R}\left(\mu_{R} \Delta+\hbar \omega_{R}\right)$, and $\Delta=(1-\epsilon) /(1+\epsilon)$.

Two-dimensional model. We first discuss the two-dimensional (2D) case with $g=$ $4 \pi \hbar^{2} m^{-1} / \ln \left(4 e^{-2 \gamma} / a^{2} \kappa^{2}\right) \ll 1 \quad[40]$, where $a$ is the two-dimensional scattering length, $\gamma \approx 0.5772$ is the Euler-Mascheroni constant, and $\kappa$ is a wave-number cutoff. A suitable value of $\kappa$ can always be found in the weakly interacting regime [2]. The repulsion (attraction) is reached for scattering lengths exponentially small (large) compared to the mean interparticle separation. The contribution arising from quantum fluctuations in Eq. (3) is ultraviolet divergent. An approach to avoid this problem is through regularization methods [41-45]. We obtain a fully analytical regularized momentum integration of Eq. (3) (see Supplemental Material [46]). So, in the ultradilute limit defined by $n a^{2} \ll 1$, the $2 \mathrm{D}$ homogeneous grand potential at zero temperature, including the mean-field contribution, gives

$$
\begin{aligned}
\frac{\Omega}{L^{2}} & =-\frac{\mu_{R}^{2}}{g_{+}}-\frac{m}{8 \pi \hbar^{2}} \mu_{R}^{2} \ln \left(\frac{\epsilon_{c}}{\sqrt{e} \mu_{R}}\right) \\
& -\frac{m}{8 \pi \hbar^{2}} \mu_{R}^{2} \Delta^{2} \ln \left(\frac{\epsilon_{c} e^{-\bar{\delta} / 2}}{\mu_{R} \Delta+2 \hbar \omega_{R}}\right),
\end{aligned}
$$

where $\bar{\delta}=(1-\sqrt{\bar{B}} / \bar{A}) /(1+\sqrt{\bar{B}} / \bar{A})$, and the low-energy cutoff $\epsilon_{c}=\hbar^{2} \kappa^{2} / m$. From Eq. (4), the density $n$ is given by

$$
\begin{aligned}
n & =\frac{2 \mu_{R}}{g_{+}}+\frac{m \mu_{R}}{4 \pi \hbar^{2}} \ln \left(\frac{\epsilon_{c}}{e \mu_{R}}\right)+\frac{m \mu_{R} \Delta^{2}}{4 \pi \hbar^{2}}\left\{\ln \left(\frac{\epsilon_{c} e^{-\bar{\delta} / 2}}{\bar{A}}\right)\right. \\
& \left.-\frac{\mu_{R} \Delta}{2 \bar{A}}\left[1+\frac{2 \Delta \bar{A} \hbar \omega_{R} \mu_{R}}{\sqrt{\bar{B}}(\bar{A}+\sqrt{\bar{B}})^{2}}\right]\right\} .
\end{aligned}
$$

The homogeneous ground-state energy density $\mathcal{E}=E / L^{2}$ also can be read as

$$
\begin{aligned}
\mathcal{E} & =\frac{1}{4} g_{+} n^{2}-\hbar \omega_{R} n+\frac{m}{32 \pi \hbar^{2}} g_{+}^{2} n^{2} \ln \left(\frac{\sqrt{e} g_{+} n}{2 \epsilon_{c}}\right) \\
& +\frac{m}{32 \pi \hbar^{2}} g_{-}^{2} n^{2} \ln \left(\frac{g_{-} n+4 \hbar \omega_{R}}{2 \epsilon_{c} e^{-\delta / 2}}\right)
\end{aligned}
$$

with $g_{-}=g(1-\epsilon), \delta=(1-\sqrt{B} / A) /(1+\sqrt{B} / A)$, $2 A=g_{-} n+4 \hbar \omega_{R}$, and $B=2 \hbar \omega_{R}\left(g_{-} n+2 \hbar \omega_{R}\right)$. Now, for simplicity, we set $m=\hbar=1$, and we consider the interesting case of weakly attractive inter- and weakly repulsive intraspecies interactions, where $1 / a_{\uparrow \downarrow} \ll \sqrt{n} \ll 1 / a$. Notice that for $\omega_{R}=0$ we recover the results employed in the study of a two-dimensional and dilute liquidlike droplet phase. We find that such a self-bound structure is present even in the presence of the Rabi frequency, however, it can be unstable. Following the lines of Ref. [2], we introduce a new energy cutoff $\tilde{\epsilon}_{c}$ including the set of coupling constants defined as $\tilde{g}=4 \pi / \ln \left(4 e^{-2 \gamma} / a^{2} \tilde{\epsilon}_{c}\right)$, and $\tilde{g}_{\uparrow \downarrow}=4 \pi / \ln \left(4 e^{-2 \gamma} / a_{\uparrow \downarrow}^{2} \tilde{\epsilon}_{c}\right)$. We choose $\tilde{\epsilon}_{c}=4 e^{-2 \gamma} / a_{\uparrow \downarrow} a$ such that $\tilde{g}_{\uparrow \downarrow}^{2}=\tilde{g}^{2}$. Provided that $\tilde{\epsilon}_{c} / \epsilon$ is not exponentially large, $g$ and $\tilde{g}$ are equivalent. Thus from Eq. (6) 


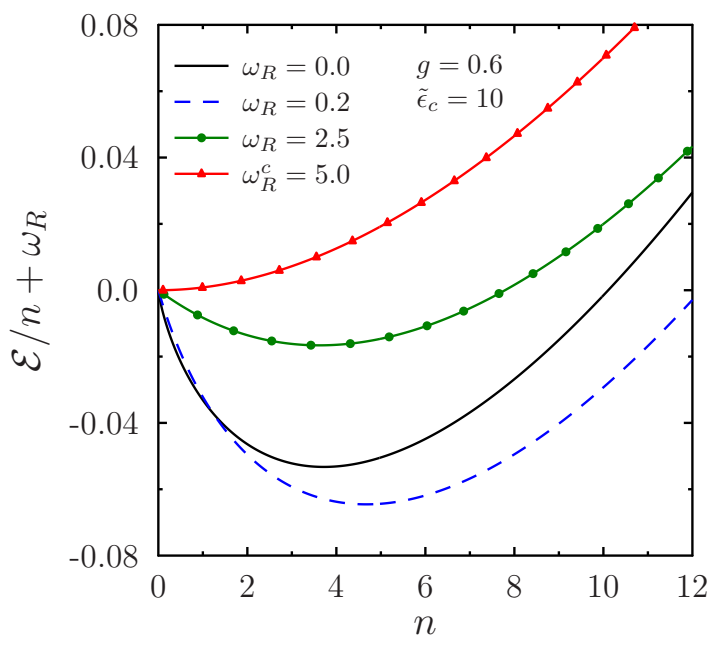

FIG. 1. The shifted energy per particle $\mathcal{E} / n+\omega_{R}$ vs the density $n$ for a two-dimensional self-bound Rabi-coupled droplet in a Bose-Bose mixture, Eq. (7). The solid black line corresponds to a droplet phase without Rabi coupling. We also take into account different values of Rabi coupling, $\omega_{R}=0.2$ (blue, dashed line), $\omega_{R}=2.5$ (green, circles), and the critical value $\omega_{R}^{c}=5.0$ (red, triangles).

the energy per particle becomes

$$
\frac{\mathcal{E}}{n}=-\omega_{R}+\frac{g^{2} n}{8 \pi} \ln \left(\frac{g n+2 \omega_{R}}{\tilde{\epsilon}_{c} e^{-\tilde{\delta} / 2}}\right),
$$

where $\tilde{\delta}=(1-\sqrt{\tilde{B}} / \tilde{A}) /(1+\sqrt{\tilde{B}} / \tilde{A}), \tilde{A}=g n+2 \omega_{R}$, and $\tilde{B}=4 \omega_{R}\left(g n+\omega_{R}\right)$. The mean-field contribution of Rabi coupling gives rise to an energy per particle shift [47, 48]. However, we show that for fixed values of energy cutoff $\tilde{\epsilon}_{c}=10$, and the coupling constant $g=0.6$, the increase of $\omega_{R}$ in the quantum correction induces a self-bound droplet instability as plotted in Fig. 1. This effect is completely missed by the mean-field approximation. We also find that the energy-cutoff dependence of Eq. (7) leads us to establish that the droplet becomes unstable for $\tilde{\epsilon}_{c} \leq 2 \omega_{R}$.

The above results apply to a purely $2 \mathrm{D}$ system. However, let us now comment briefly on the applicability of these in quasi-2D mixtures. We analyze the case of a harmonic confinement. In a quasi-2D system we have $a=2 l_{0} \sqrt{\pi / B} e^{-\sqrt{\pi / 2} l_{0} / a^{(3 D)}-\gamma}[40]$, with $a^{(3 D)}$ the threedimensional scattering length, $l_{0}$ the oscillator length of the trap in the confinement direction, and $B \approx 0.9$. So, the weakly interacting quasi-2D regime is reached as long as $0<-a_{\uparrow \downarrow}^{(3 D)}<a^{(3 D)} \ll l_{0}[2]$.

One-dimensional model. We now turn to the weakly interacting one-dimensional scenario, which requires $|g| / n \ll 1$. In particular, the $1 \mathrm{D}$ interparticle interaction can be well approximated by an effective coupling constant $g=-2 \hbar^{2} / m a$ [49], with $a$ the $1 \mathrm{D}$ scattering length. In an attempt to get an analytical solution of Eq. (3), and thus insights of the underlying physics, we consider the limit of small Rabi coupling. Then, by ex- panding up to the linear term in the Rabi frequency, we find that Eq. (3) presents ultraviolet divergences, and an appropriate modification is necessary to cure these [41-45]. We employ dimensional regularization (see Supplemental Material [46]). In this way, the regularized and homogeneous grand potential at zero temperature, including the mean-field contribution, is

$$
\begin{aligned}
\frac{\Omega}{L} & =-\frac{\mu_{R}^{2}}{g_{+}}-\frac{2}{3 \pi}\left(\frac{m}{\hbar^{2}}\right)^{1 / 2} \mu_{R}^{3 / 2}-\frac{2}{3 \pi}\left(\frac{m}{\hbar^{2}}\right)^{1 / 2}(\mu \Delta)^{3 / 2} \\
& -\frac{\omega_{R}}{\pi}(m \mu \Delta)^{1 / 2}\left[\Delta+\frac{1}{2} \ln \left(\frac{\epsilon_{c}}{\mu \Delta}\right)\right]
\end{aligned}
$$

where $\epsilon_{c} \equiv \hbar^{2} \kappa^{2} e^{4} / 64 m$, with $\kappa$ an arbitrary wavenumber scale or renormalization scale parameter. The logarithmic contribution, although unusual in a 1D model, is proper for the regularization of the integrals provided by Eq. (3), and its effect is discussed later. The respective one-dimensional density is written as

$$
\begin{aligned}
n & =\frac{2 \mu_{R}}{g_{+}}+\frac{1}{\pi}\left(\frac{m}{\hbar^{2}}\right)^{1 / 2}\left(\mu_{R}^{1 / 2}+\Delta^{3 / 2} \mu^{1 / 2}\right) \\
& +\frac{\omega_{R}}{2 \pi} \sqrt{\frac{m \Delta}{\mu}}\left[\Delta+\frac{1}{2} \ln \left(\frac{\epsilon_{c}}{e \mu \Delta}\right)\right],
\end{aligned}
$$

and the ground-state energy density is given by

$$
\begin{aligned}
\mathcal{E} & =\frac{1}{4} g_{+} n^{2}-\hbar \omega_{R} n-\frac{1}{3 \pi}\left(\frac{m}{2 \hbar^{2}}\right)^{1 / 2} n^{3 / 2}\left(g_{+}^{3 / 2}+g_{-}^{3 / 2}\right) \\
& -\frac{\omega_{R}}{\sqrt{2} \pi}\left(m g_{-} n\right)^{1 / 2}\left[\Delta+\frac{1}{2} \ln \left(\frac{2 \epsilon_{c}}{g_{-} n}\right)\right] .
\end{aligned}
$$

From a mean-field viewpoint, and in the absence of Rabi coupling, the condition $0<\epsilon<1$ is employed in order to avoid phase separation $[50,51]$. However, for $\epsilon>1$, the inclusion of Rabi coupling gives rise to an effective attraction between the species, which can drive the immiscible configuration into a miscible state [52]. Instead, from energy (10), we find that for repulsive intraspecies and interspecies interactions $\left(g, g_{\uparrow \downarrow}>0\right)$ with $\epsilon>1$, the quantum corrections destabilize the system. On the other hand, in the regime of repulsive $(g>0)$ intraspecies and attractive $\left(g_{\uparrow \downarrow}<0\right)$ interspecies interactions for $\epsilon<-1$, the system becomes fully attractive and unstable, contrasting thus the three-dimensional one [31]. In such a 3D scenario, the resulting energy density displays an interplay between an attractive mean-field term $\propto-n^{2}$ and a repulsive beyond-mean-field correction $\propto n^{5 / 2}$. This feature opens the door to the possibility of observing a droplet phase. We now focus on the regime of repulsive intraspecies and attractive interspecies interactions with $|\epsilon| \sim 1[2]$. By using $g=2 \hbar^{2} / m|a|, g_{\uparrow \downarrow}=-2 \hbar^{2} / m\left|a_{\uparrow \downarrow}\right|$ [49], and by taking $E_{B}=\hbar^{2} / m|a|^{2}$ as the energy unit, we define $\bar{n}=n|a|, \bar{\omega}_{R}=\hbar \omega_{R} / E_{B}$, and $\bar{\epsilon}_{c}=\epsilon_{c} / E_{B}$. Thus we get the scaled energy per particle of ultradilute and uniform Rabi-coupled mixtures $\overline{\mathcal{E}} / \bar{n}=\mathcal{E} /\left(\bar{n} E_{B} /\left|a^{1 D}\right|\right)$ as

$$
\frac{\overline{\mathcal{E}}}{\bar{n}}=\frac{1}{2} \epsilon_{-} \bar{n}-\bar{\omega}_{R}-\frac{4 \sqrt{2}}{3 \pi} \bar{n}^{1 / 2}-\frac{2 \sqrt{2}}{\pi} \frac{\bar{\omega}_{R}}{\epsilon_{-} \bar{n}^{1 / 2}},
$$




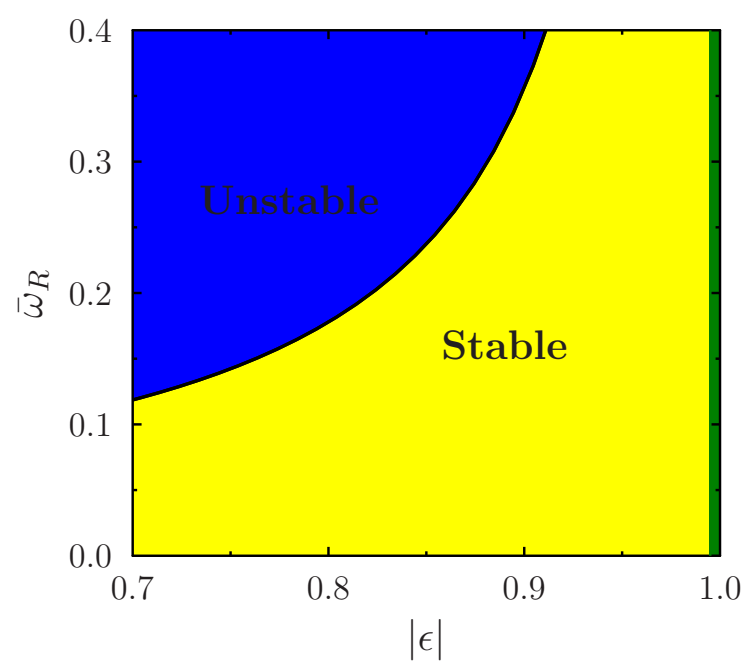

FIG. 2. Stability diagram of one-dimensional self-bound Rabi-coupled droplets in Bose-Bose mixtures. The green band shows the asymptotic behavior $|\epsilon| \rightarrow 1$.

where $\epsilon_{-}=1-|\epsilon| \rightarrow 0$. In this regime, we find that

$$
\frac{1}{\epsilon_{-}} \gg \frac{1}{4} \ln \left(\frac{\overline{\epsilon_{c}}}{4 \bar{n}}\right) \text {. }
$$

So, we check that the logarithmic contribution of energy (10) does not affect the results, and hence it is neglected [53]. This expression accounts for the beyond-mean-field attractive correction to the ground-state energy. In the absence of Rabi coupling such a mixture gets into a pure dilute liquidlike droplet state. Here, we find that such energy per particle with $\bar{\omega}_{R} \neq 0$ displays that the Rabi coupling shifts the ground-state energy of the self-bound droplet, and also it leads to the possibility of obtaining a quantum mechanical instability of the mixture. By minimizing Eq. (11) with respect to density, we find two possible physical solutions which are written as

$$
\bar{n}_{j}=\frac{128}{81 \pi^{2} \epsilon_{-}^{2}}\left[\cos \left[\frac{1}{3} \cos ^{-1} \theta\left(\bar{\omega}_{R},|\epsilon|\right)-\frac{2 \pi}{3} j\right]+\frac{1}{2}\right]^{2}(13)
$$

where $\theta=1-729 \pi^{2} \bar{\omega}_{R} \epsilon_{-} / 128$, and $j=0,1$. For $j=0$ we have the equilibrium density, hereafter called $\bar{n}_{0}$, while for $j=1$ the solution is a local maximum. From $\bar{n}_{0}$ we find a stable mixture for $0<\bar{\omega}_{R}(1-|\epsilon|) \leq 256 / 729 \pi^{2}$, as plotted in Fig. 2. The green band shows the asymptotic behavior $|\epsilon| \rightarrow 1$. This diagram could be understood as a result of the combined attraction-repulsion effect of the different contributions in Eq. (11). As a particular case we display in Fig. 3 the energy per particle given by Eq. (11) as a function of the density. We consider a fixed value of $|\epsilon|=0.9$. The increase of $\bar{\omega}_{R}$ shows as the local minimum disappears when this frequency exceeds the critical value of $\bar{\omega}_{R}^{c} \simeq 0.356$, as predicted by Eq. (13). Beyond this critical parameter we have an effective attraction between atoms and the ground state becomes unstable. This is a similar mechanism to the instability by collapse in a single-component 3D BEC

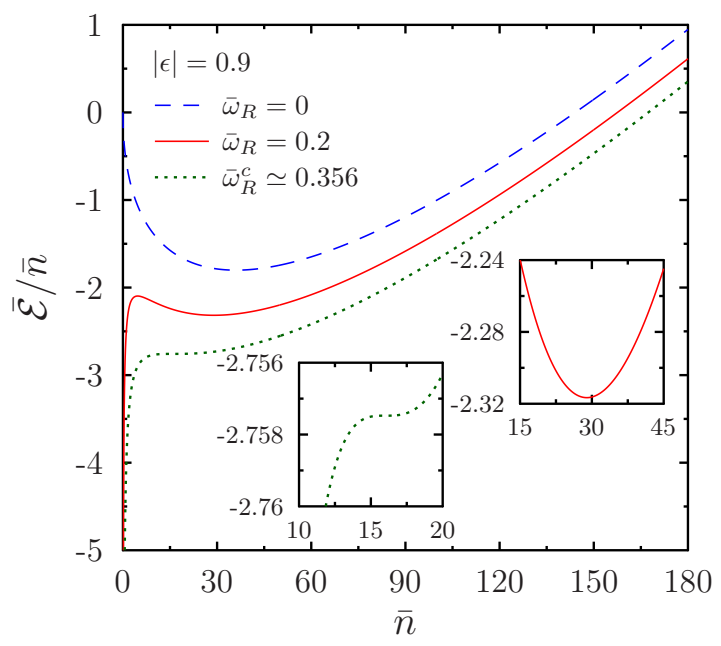

FIG. 3. The energy per particle $\overline{\mathcal{E}} / \bar{n}$ vs the density $\bar{n}$ for an one-dimensional Rabi-coupled Bose-Bose mixture, Eq. (11). The dashed line (blue) represents the droplet phase in the absence of Rabi coupling. The solid line (red) considers $\bar{\omega}_{R}=$ 0.2 . The dotted line (green) corresponds to the critical value $\bar{\omega}_{R}^{c} \simeq 0.356$ at which the system becomes unstable. Inset: We present the local minimum localization of $\overline{\mathcal{E}} / \bar{n}$ and predicted by Eq. (13).

$[50,51]$. We also calculate the spinodal region of the mixture $\bar{n}^{\mathrm{sp}}$, which is defined by the condition $\partial^{2} \overline{\mathcal{E}} / \partial \bar{n}^{2}=$ 0 . This spinodal density has the same form of Eq. (13), provided that $\epsilon_{-} \rightarrow 4 \epsilon_{-} / 3$ and $\omega_{R} \rightarrow 4 \omega_{R} / 9$. So, the mixture is thus metastable for $\bar{n}^{\mathrm{sp}}<\bar{n}<\bar{n}_{0}$. In the case of $|\epsilon|=0.9$ and $\omega_{R}=0.2$, we find a metastable mixture for $18.085<\bar{n}<28.945$. From the ground-state energy density of the Rabi-coupled droplet, Eq. (11), we also get some relevant quantities as the equilibrium chemical potential $\bar{\mu}_{0}=\bar{\mu}\left(\bar{n}=\bar{n}_{0}\right)$,

$$
\bar{\mu}_{0}=\epsilon_{-} \bar{n}_{0}-\bar{\omega}_{R}-\frac{2 \sqrt{2}}{\pi} \bar{n}_{0}^{1 / 2}-\frac{\sqrt{2}}{\pi} \frac{\bar{\omega}_{R}}{\epsilon_{-} \bar{n}_{0}^{1 / 2}},
$$

and the corresponding scaled speed of sound $\bar{c}_{0} \equiv$ $c_{0} / \sqrt{E_{B} / m}$, given by

$$
\bar{c}_{0}=\left(\epsilon_{-} \bar{n}_{0}-\frac{\sqrt{2}}{\pi} \bar{n}_{0}^{1 / 2}+\frac{1}{\sqrt{2} \pi} \frac{\bar{\omega}_{R}}{\epsilon_{-} \bar{n}_{0}^{1 / 2}}\right)^{1 / 2} .
$$

Let us now comment on the applicability of these results in quasi-1D mixtures. In a quasi-1D system where a harmonic radial confinement with oscillator length $l_{\perp}$ is assumed, the relation between $a^{(3 D)}$ and the one-dimensional scattering length is $a=-l_{\perp}\left(l_{\perp}-\right.$ $\left.C a^{(3 D)}\right) / a^{(3 D)}[49]$, with $C \simeq 1.0326$. So, an effective weakly interacting quasi-1D regime is obtained as long as the radial confinement, fixed by the radial oscillator length, is much greater than the three-dimensional scattering lengths, i.e., $l_{\perp} \gg a^{(3 D)}>-a_{\uparrow \downarrow}^{(3 D)}>0$.

Conclusion. We study ultracold and ultradilute weakly interacting low-dimensional Rabi-coupled BoseBose mixtures beyond the mean-field approximation. In 
particular, we focus on the case where the interspecies interactions are weakly attractive and the intraspecies ones are weakly repulsive. Our results show that in these regimes such mixtures manifest the formation of a liquidlike Rabi-coupled droplet phase including a quantum instability encoded in the Rabi frequency. Remarkably, in the $1 \mathrm{D}$ mixture we provide an analytical equilibrium density, and also identify the spinodal region of the selfbound droplets. Furthermore, in the stable liquidlike state, we also calculate the chemical potential, and the speed of sound. The above general features of Rabicoupled droplets are directly linked to the crucial nature of the quantum fluctuations, and hence may stimulate and play an interesting role in future experiments. It also may be interesting to verify the validity of our results by means of the recent developments on quantum Monte Carlo methods [22, 23].
[1] D. S. Petrov, Quantum mechanical stabilization of a collapsing Bose-Bose mixture, Phys. Rev. Lett. 115, 155302 (2015).

[2] D. S. Petrov and G. E. Astrakharchik, Ultradilute lowdimensional liquids, Phys. Rev. Lett. 117, 100401 (2016).

[3] H. Kadau, M. Schmitt, M. Wenzel, C. Wink, T. Maier, I. Ferrier-Barbut, and T. Pfau, Observing the Rosensweig instability of a quantum ferrofluid, Nature (London) 530, 194 (2016).

[4] M. Schmitt, M. Wenzel, F. Böttcher, I. Ferrier-Barbut, and T. Pfau, Self-bound droplets of a dilute magnetic quantum liquid, Nature (London) 539, 259 (2016).

[5] I. Ferrier-Barbut, H. Kadau, M. Schmitt, M. Wenzel, and T. Pfau, Observation of quantum droplets in a strongly dipolar Bose gas, Phys. Rev. Lett. 116, 215301 (2016).

[6] I. Ferrier-Barbut, M. Schmitt, M. Wenzel, H. Kadau, and T. Pfau, Liquid quantum droplets of ultracold magnetic atoms, J. Phys. B: At. Mol. Opt. Phys. 49214004 (2016).

[7] L. Chomaz, S. Baier, D. Petter, M. J. Mark, F. Wächtler, L. Santos, and F. Ferlaino, Quantum-fluctuation-driven crossover from a dilute Bose-Einstein condensate to a macro-droplet in a dipolar quantum fluid, Phys. Rev. X 6, 041039 (2016).

[8] C. R. Cabrera, L. Tanzi, J. Sanz, B. Naylor, P. Thomas, P. Cheiney, and L. Tarruell, Quantum liquid droplets in a mixture of Bose-Einstein condensates, Science 359, 301 (2018).

[9] G. Semeghini, G. Ferioli, L. Masi, C. Mazzinghi, L. Wolswijk, F. Minardi, M. Modugno, G. Modugno, M. Inguscio, and M. Fattori, Self-bound quantum droplets of atomic mixtures in free space, Phys. Rev. Lett. 120, 235301 (2018).

[10] P. Cheiney, C. R. Cabrera, J. Sanz, B. Naylor, L. Tanzi, and L. Tarruell, Bright soliton to quantum droplet transition in a mixture of Bose-Einstein condensates, Phys. Rev. Lett. 120, 135301 (2018).

[11] T. D. Lee and C. N. Yang, Quantum-mechanical manybody problem with hard-sphere interaction, Phys. Rev. 105, 1119 (1957).

[12] T. D. Lee, K. Huang, and C. N. Yang, Eigenvalues and eigenfunctions of a Bose system of hard spheres and its low-temperature properties, Phys. Rev. 106, 1135 (1957).

[13] K.T. Xi and H. Saito, Droplet formation in a BoseEinstein condensate with strong dipole-dipole interaction, Phys. Rev. A 93, 011604(R) (2016).

[14] P. B. Blakie, Properties of a dipolar condensate with three-body interactions, Phys. Rev. A 93, 033644 (2016).

[15] F. Wächtler and L. Santos, Quantum filaments in dipolar Bose-Einstein condensates, Phys. Rev. A 93, 061603(R) (2016).
[16] F. Wächtler and L. Santos, Ground-state properties and elementary excitations of quantum droplets in dipolar Bose-Einstein condensates, Phys. Rev. A 94, 043618 (2016).

[17] D. Baillie, R. M. Wilson, R. N. Bisset, and P. B. Blakie, Self-bound dipolar droplet: a localized matter wave in free space, Phys. Rev. A 94, 021602(R) (2016).

[18] R. N. Bisset, R. M. Wilson, D. Baillie, and P. B. Blakie, Ground-state phase diagram of a dipolar condensate with quantum fluctuations, Phys. Rev. A 94, 033619 (2016).

[19] H. Saito, Path-integral Monte Carlo study on a droplet of a dipolar Bose-Einstein condensate stabilized by quantum fluctuation, J. Phys. Soc. Jpn. 85, 053001 (2016).

[20] A. Macia, J. Sánchez-Baena, J. Boronat, and F. Mazzanti, Droplets of trapped quantum dipolar bosons, Phys. Rev. Lett. 117, 205301 (2016).

[21] F. Cinti, A. Cappellaro, L. Salasnich, and T. Macrì, Superfluid filaments of dipolar bosons in free space, Phys. Rev. Lett. 119, 215302 (2017).

[22] V. Cikojević, K. Dželalija, P. Stipanović, L. Vranješ Markić, and J. Boronat, Ultradilute quantum liquid drops, Phys. Rev. B 97, 140502(R) (2018).

[23] L. Parisi, G.E. Astrakharchik, and S. Giorgini, Liquid state of one-dimensional Bose mixtures: a quantum Monte Carlo study, Phys. Rev. Lett. 122, 105302 (2019).

[24] E. Chiquillo, Equation of state of the one- and threedimensional Bose-Bose gases, Phys. Rev. A 97, 063605 (2018).

[25] Y. J. Lin, K. Jiménez-García, and I. B. Spielman, Spinorbit-coupled Bose-Einstein condensates, Nature (London) 471, 83 (2011).

[26] J. Y. Zhang, S. C. Ji, Z. Chen, L. Zhang, Z. D. Du, B. Yan, G. S. Pan, B. Zhao, Y. J. Deng, H. Zhai, S. Chen, and J. W. Pan, Collective dipole oscillation of a spin-orbit coupled Bose-Einstein condensate, Phys. Rev. Lett. 109, 115301 (2012).

[27] C. Wang, C. Gao, C.M. Jian, and H. Zhai, Spin-orbit coupled spinor Bose-Einstein condensates, Phys. Rev. Lett. 105, 160403 (2010).

[28] T.L. Ho and S. Zhang, Bose-Einstein condensates with spin-orbit interaction, Phys. Rev. Lett. 107, 150403 (2011)

[29] Y. Li, L. P. Pitaevskii, and S. Stringari, Quantum tricriticality and phase transitions in spin-orbit coupled Bose-Einstein condensates, Phys. Rev. Lett. 108, 225301 (2012).

[30] A. Manchon, H. C. Koo, J. Nitta, S. M. Frolov, and R. A. Duine, New perspectives for Rashba spin-orbit coupling, Nat. Mater. 14, 871 (2015).

[31] A. Cappellaro, T. Macrì, G. F. Bertacco and L. Salasnich, 
Equation of state and self-bound droplet in Rabi-coupled Bose mixtures, Sci. Rep. 7, 13358 (2017).

[32] D. T. Son and M. A. Stephanov, Domain walls of relative phase in two-component Bose-Einstein condensates, Phys. Rev. A 65, 063621 (2002).

[33] C. P. Search, A. G. Rojo, and P. R. Berman, Ground state and quasiparticle spectrum of a two-component Bose-Einstein condensate, Phys. Rev. A 64, 013615 (2001).

[34] M. Abad and A. Recati, A study of coherently coupled two-component Bose-Einstein condensates, Eur. Phys. J. D 67, 40053, (2013).

[35] D. S. Petrov, D. M. Gangardt, and G. V. Shlyapnikov, Low-dimensional trapped gases, J. Phys. IV (France) 116, 5 (2004).

[36] Y. Castin, Simple theoretical tools for low dimension Bose gases, J. Phys. IV (France) 116, 89 (2004).

[37] J. Armaitis, H. T. C. Stoof, and R. A. Duine, Hydrodynamic modes of partially condensed Bose mixtures, Phys. Rev. A 91, 043641 (2015).

[38] J.O. Andersen, Theory of the weakly interacting Bose gas, Rev. Mod. Phys. 76, 599 (2004).

[39] H.T.C Stoof, K.B. Gubbels, and D.B.M. Dickerscheid, Ultracold Quantum Fields (Springer, Berlin, 2009).

[40] D.S. Petrov and G.V. Shlyapnikov, Interatomic collisions in a tightly confined Bose gas, Phys. Rev. A 64, 012706 (2001).

[41] 't Hooft, G. and M. Veltman, Regularization and renormalization of gauge fields, Nucl. Phys. B 44, 189 (1972).

[42] H. Kleinert and V. Schulte-Frohlinde, Critical Properties of $\phi^{4}$-Theories, (World Scientific, Singapore, 2001).

[43] J.O. Andersen, Ground state pressure and energy density of a homogeneous Bose gas in two dimensions, Eur. Phys. J B 28, 389 (2002).

[44] C. Mora and Y. Castin, Extension of Bogoliubov theory to quasicondensates, Phys. Rev. A 67, 053615 (2003).

[45] L. Salasnich and F. Toigo, Zero-point energy of ultracold atoms, Phys. Rep. 640, 1 (2016).

[46] See Supplemental Material for more details on the nontrivial regularization of the grand potential in two and one dimensions.

[47] E. Chiquillo, Harmonically trapped attractive and repulsive spin-orbit and Rabi-coupled Bose-Einstein condensates, J. Phys. A 50, 105001 (2017).

[48] E. Chiquillo, Quasi-one-dimensional spin-orbit- and Rabi-coupled bright dipolar Bose-Einstein-condensate solitons, Phys. Rev. A 97, 013614 (2018).

[49] M. Olshanii, Atomic scattering in the presence of an external confinement and a gas of impenetrable Bosons, Phys. Rev. Lett. 81, 938 (1998).

[50] L. P. Pitaevskii and S. Stringari, Bose-Einstein Condensation and Superfluidity (Clarendon, Oxford, UK, 2016).

[51] C. J. Pethick and H. Smith, Bose-Einstein Condensate in Dilute Gases, (Cambridge, University Press, UK, 2008).

[52] I. M. Merhasin, B. A. Malomed, and R. Driben, Transition to miscibility in a binary Bose-Einstein condensate induced by linear coupling, J. Phys. B 38, 877 (2005).

[53] Regarding the energy cutoff in the logarithmic term, we also consider the scaled energy per particle in a general context. We find that for $0<\epsilon \ll 1$, the mean-field contribution dominates over the quantum corrections. So, the effect of $\bar{\epsilon}_{c}$ is not relevant either. We confirm our results by using $3 \cdot 10^{-1} \leq \bar{\epsilon}_{c} \leq 5 \cdot 10^{3}$. 


\section{SUPPLEMENTAL MATERIAL}

Grand potential in two dimensions. From Eq. (3) in main text, the grand potential in two dimensions takes the form

$$
\frac{\Omega_{g}}{L^{2}}=I_{a}+I_{b}
$$

where,

$$
I_{a}=\int_{0}^{\infty} \frac{d k}{4 \pi} k \sqrt{\frac{\hbar^{2} k^{2}}{2 m}\left(\frac{\hbar^{2} k^{2}}{2 m}+2 \mu_{R}\right)},
$$

and

$$
I_{b}=\int_{0}^{\infty} \frac{d k}{4 \pi} k \sqrt{\frac{\hbar^{2} k^{2}}{2 m}\left(\frac{\hbar^{2} k^{2}}{2 m}+2 \bar{A}\right)+\bar{B}} .
$$

To calculate $I_{a}$, we employ dimensional regularization in the modified minimal subtraction scheme $\overline{\mathrm{MS}}$-scheme. In a similar way, this integral also is calculated by means of convergence-factor regularization (CFR) [1], with same results. To deal with the second term we use the CFR method. Thus, $I_{a}$ is extended to a noninteger and generic $D=2-2 \bar{\varepsilon}$ dimension, and the limit $\bar{\varepsilon} \rightarrow 0$ is applied at the end of the calculation. So

$$
\begin{aligned}
I_{a} & =-\frac{m \mu_{R}^{2}}{4 \pi^{3 / 2} \hbar^{2}}\left(\frac{\hbar^{2} \pi}{m \mu_{R}}\right)^{\bar{\varepsilon}}\left(\frac{e^{\gamma} \kappa^{2}}{4 \pi}\right)^{\bar{\varepsilon}} \Gamma\left(\frac{3}{2}\right) \\
& \times\left[\frac{1}{\bar{\varepsilon}}+\ln \left(\frac{4}{e^{\gamma+1 / 2}}\right)\right],
\end{aligned}
$$

where $\kappa$ is an arbitrary wavenumber scale or renormalization scale parameter. The factor $\left(e^{\gamma / 4 \pi}\right)^{\bar{\varepsilon}}$ is introduced so that, after minimal subtraction of the poles in $\bar{\varepsilon}, \kappa$ coincides with the renormalization scale of the $\overline{\mathrm{MS}}$-scheme [2]. Then by expanding $I_{a}$ in terms of the parameter $\bar{\varepsilon}$, we get

$$
I_{a}=-\frac{m \mu_{R}^{2}}{8 \pi \hbar^{2}}\left[\frac{1}{\bar{\varepsilon}}+\ln \left(\frac{\hbar^{2} \kappa^{2}}{m \mu_{R} \sqrt{e}}\right)+\mathcal{O}(\bar{\varepsilon})\right] .
$$

By performing the appropriate counterterms subtraction to remove the pole in $\bar{\varepsilon}[3-7]$, and by using the limit $\bar{\varepsilon} \rightarrow 0$, we obtain the second term in Eq. (4) of main text.

Regarding to $I_{b}$, we have

$$
\begin{aligned}
I_{b} & =\frac{m}{2 \pi \hbar^{2}} \int d z\left[z \sqrt{z^{4}+2 \bar{A} z^{2}+\bar{B}}\right. \\
& \left.-\left(z^{3}+\bar{A} z+\frac{\bar{B}-\bar{A}^{2}}{2 z}\right)\right]
\end{aligned}
$$

with $z^{2}=\hbar^{2} k^{2} / 2 m$. The counterterms are determined by expanding the Rabi excitation $E_{b}$ at high momentum [8]. Solution of this integral is given by the last term in Eq. (4) of main text.

Grand potential in one dimension. In the onedimensional case and employing the approximation of small Rabi-coupling, Eq. (3) of main text is written as

$$
\frac{\Omega_{g}}{L}=I_{a}+I_{1}+I_{2}+I_{3}
$$

where the integrals $I_{a}, I_{1}$, and $I_{2}$ are calculated by means of dimensional regularization (with same results using CFR). Thus

$$
\begin{aligned}
I_{a} & =\int_{0}^{\infty} \frac{d k}{2 \pi} \sqrt{\frac{\hbar^{2} k^{2}}{2 m}\left(\frac{\hbar^{2} k^{2}}{2 m}+2 \mu_{R}\right)} \\
& =-\frac{2}{3 \pi}\left(\frac{m}{\hbar^{2}}\right)^{1 / 2} \mu_{R}^{3 / 2}, \\
I_{1} & =\int_{0}^{\infty} \frac{d k}{2 \pi} \sqrt{\frac{\hbar^{2} k^{2}}{2 m}\left(\frac{\hbar^{2} k^{2}}{2 m}+2 \mu \Delta\right)} \\
& =-\frac{2}{3 \pi}\left(\frac{m}{\hbar^{2}}\right)^{1 / 2}(\mu \Delta)^{3 / 2},
\end{aligned}
$$

and,

$$
\begin{aligned}
I_{2} & =\frac{1}{\pi} \omega_{R}(\Delta+2)(m \mu \Delta)^{1 / 2} \int_{0}^{\infty} \frac{x}{\sqrt{x^{2}+1}} \\
& =-\frac{\omega_{R}}{\pi}(\Delta+2)(m \mu \Delta)^{1 / 2} .
\end{aligned}
$$

with $x^{2}=\hbar^{2} k^{2} / 4 m \mu \Delta$. Integration over momentum of $I_{3}$ it is still divergent, so we also use the $\overline{\mathrm{MS}}$-scheme. Then through $D=1-2 \bar{\varepsilon}$, we get

$$
\begin{aligned}
I_{3} & =\frac{1}{\pi} \omega_{R}(m \mu \Delta)^{1 / 2} \int_{0}^{\infty} \frac{1}{x \sqrt{x^{2}+1}} \\
& =\frac{\omega_{R}}{2 \pi}(m \mu \Delta)^{1 / 2}\left(\frac{\pi \hbar^{2}}{m \mu \Delta}\right)^{\bar{\varepsilon}}\left(\frac{e^{\gamma} \kappa^{2}}{4 \pi}\right)^{\bar{\varepsilon}} \frac{\Gamma(-\bar{\varepsilon}) \Gamma\left(\frac{1}{2}+\bar{\varepsilon}\right)}{\Gamma\left(\frac{1}{2}-\bar{\varepsilon}\right)} \\
& =\frac{\omega_{R}}{2 \pi}(m \mu \Delta)^{1 / 2}\left[-\frac{1}{\bar{\varepsilon}}+\ln \left(\frac{64 m \mu \Delta}{\hbar^{2} \kappa^{2}}\right)\right]+\mathcal{O}(\bar{\varepsilon})
\end{aligned}
$$

By taking into account the appropriate counterterms subtraction to remove the pole in $\bar{\varepsilon}$, and applying the limit $\bar{\varepsilon} \rightarrow 0$, we obtain the last term of Eq. (8) in main text. $I_{3}$ also is solved by means of CFR and we find equivalence between both methods as long as $\kappa_{\mathrm{MS}}^{2}=4 q_{\mathrm{CFR}}^{2}$, with $q_{\mathrm{CFR}}$ a low-wavenumber cutoff.
[1] L. Salasnich and F. Toigo, Phys. Rep. 640, 1 (2016).

[2] H. Kleinert and V. Schulte-Frohlinde, "Critical Properties of $\phi^{4}$-Theories", (World Scientific, Singapore, 2001).

[3] T. Haugset, H. Haugerud, and F. Ravndal, Ann. of Phys. 266, 27 (1998).

[4] E. Braaten and A. Nieto, Eur. Phys. J. B 11, 143 (1999).
[5] P. Arnold, G. Moore, and B. Tomášik, Phys. Rev. A 65, 013606 (2001).

[6] J.O. Andersen, Eur. Phys. J B 28, 382 (2002).

[7] P. A. and J.O. Andersen, Phys. Rev. D 95, 036009 (2017).

[8] A. Cappellaro, T. Macrì, G. F. Bertacco and L. Salasnich, Sci. Rep. 7, 13358 (2017). 